\title{
HÉRNIA DE BOCHDALEK EM ADULTO
}

\section{BOCHDALEK HERNIA IN ADULT}

\section{Daniel Riccioppo C.F. de Oliveira, ACBC-SP1' Aldo Junqueira Rodrigues Jr., TCBC-SP²}

\section{INTRODUÇÃO}

A primeira hérnia diafragmática foi descrita por Ambroise Paré, em 1575. A hérnia de Bochdalek (HB) é defeito congênito situado na porção posterolateral do diafragma por obliteração incompleta de forame situado nesta região. Foi descrita, primeiramente, pelo professor de anatomia Bochdalek, em 1848, com relato de dois casos cuja etiologia foi atribuída à ruptura da membrana do trígono lombocostal. Em 1902, o primeiro caso de correção cirúrgica bem sucedida foi realizado por Heidenhain em paciente de 19 anos ${ }^{1,2,3}$.

Sua apresentação ocorre normalmente na infância, com incidência em neonatos, variando de 1:4000 a 1:7000. O diagnóstico nas primeiras oito semanas de vida representa 5 a $25 \%$ de todas as $\mathrm{HB}$, um dos defeitos congênitos do tórax mais comuns. É causa de insuficiência respiratória aguda no neonato, com índices de mortalidade descritos, em 1965, entre 35 e 40\%, mantendo-se nestes níveis em $1996^{4,5}$.

No adulto é achado raro, com pouco mais de 100 casos descritos. Com incidência entre $1 \%$ e $5 \%$, representa aproximadamente 5\% das HB. Em estudo retrospectivo de mais de 13000 CT abdominais, em 1998, foi achado incidental em $0,17 \%$ dos exames ${ }^{6,7,8}$. Ocorre predominantemente em mulheres, com relação de 17 mulheres para cada cinco homens $(77 \%)$, principalmente na sexta década de vida, variando em relatos de 23 a 91 anos de idade ${ }^{2,6}$.

Usualmente, apresenta-se no adulto por sintomas relacionados a complicações decorrentes de vísceras abdominais herniadas, com encarceramento, estrangulamento, sofrimento e perfuração em nível torácico ou abdominal com suas conseqüentes intercorrências sépticas. O efeito de massa no tórax pode causar alterações anatômicas e fisiológicas no sistema cardio-respiratório, decorrentes da compressão de estruturas aí existentes. Apresenta-se como manifestação aguda em 34 a $46 \%$ das casuísticas publicadas, com mortalidade relatada em $32 \%$ em vigência de estrangulamento ${ }^{2,7,8}$. Quando tratada eletivamente a letalidade cai para 3\%9.

Quanto aos sintomas relacionados com sua presença, estes podem apresentar-se como agudos ou recorrentes, devendo esta anomalia entrar no diagnóstico diferencial de abdome agudo ou dor abdominal.

Ilustrativamente apresentamos relato de caso de HB em paciente adulto com sintomas digestivos recidivantes, com posterior revisão da literatura.

\section{HÉRNIA DE BOCHDALEK NO ADULTO}

Paciente do sexo feminino, 49 anos, portadora de Síndrome de Sjögren em uso de corticoterapia, procura o ambulatório com queixa de epigastralgia pós-prandial de longa data. Há mais de cinco anos refere quadro de dor epigástrica com início pós prandial, que melhorava espontaneamente após algumas horas. Relatava alívio parcial com analgésicos comuns, mas não com antiácidos. Negava vômitos, disfagia ou tosse. Sem história prévia de trauma torácico ou abdominal ou cirurgias prévias.

Ao exame físico a paciente apresentava-se eutrófica, em bom estado geral, corada e hidratada. Não havia dor à palpação abdominal e os ruídos hidro-aéreos eram normais. A propedêutica pulmonar e cardíaca era normal.

Feita Endoscopia digestiva alta, esta mostrou ausência de hérnia hiatal ou de sinais inflamatórios ou de lesões em esôfago, estômago ou duodeno. À retrovisão observou-se herniação do fundo gástrico com mucosa normal em anel diafragmático evidente (Figura 1). Tomografia Computadorizada de tórax e abdome confirmou hérnia diafragmática de posterolateral à esquerda (Figura 2).

A paciente foi então submetida à correção cirúrgica de hérnia de Bochdalek utilizando-se uma toracotomia lateral esquerda no sétimo espaço intercostal, com ressecção parcial da sexta costela. Identificado o problema verificouse a presença de uma massa de consistência fibroelástica aderida sobre o peritôneo que envolvia as estruturas abdominais herniadas, medindo $11,0 \times 3,0 \times 2,0 \mathrm{~cm}$. Aberto o saco herniário identificou-se o fundo gástrico já na cavidade abdominal (Figura 3). Ressecada a massa aderida ao saco herniário, que apresentava aspecto cístico com tecido pulmonar em seu interior. Realizou-se o reparo do defeito

1. Cirurgião Assistente da Unidade de Estômago, Esôfago e Duodeno, Disciplina de Gastroenterologia Cirúrgica, Escola Paulista de Medicina, Universidade Federal de São Paulo; Ex-Cirurgião Assistente da Unidade de Cirurgia Geral, Instituto do Coração do Hospital das Clínicas, Faculdade de Medicina da Universidade de São Paulo.

2. Professor Titular do Departamento de Anatomia Topográfica e Seccional, Faculdade de Medicina da Universidade de São Paulo; Diretor da Unidade de Cirurgia Geral do Instituto do Coração do Hospital das Clínicas, Faculdade de Medicina da Universidade de São Paulo.

Recebido em 20/02/2007

Aceito para publicação em 23/04/2007

Conflito de interesses: nenhum

Fonte de financiamento: nenhuma

Trabalho realizado na Unidade de Cirurgia Geral do Instituto do Coração do Hospital das Clínicas, Faculdade de Medicina da Universidade de São Paulo - São Paulo - SP. 


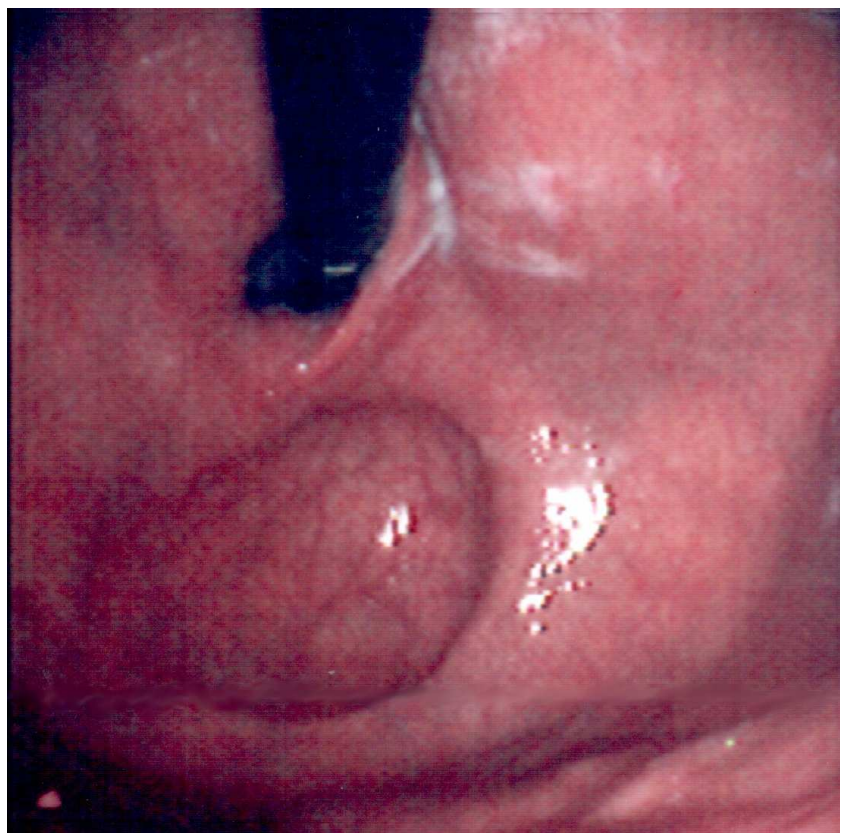

Figura 1 - Gastroscopia em manobra de retrovisão mostrando fundo gástrico com mucosa normal herniado em anel diafragmático evidente.

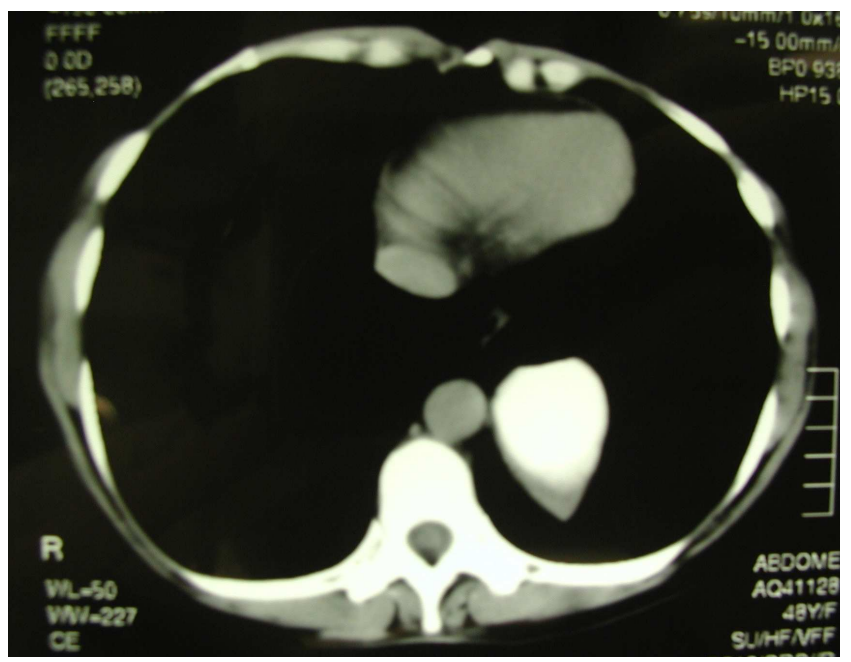

Figura 2 - Tomografia Computadorizada de tórax evidenciando hérnia diafragmática posterolateral à esquerda.

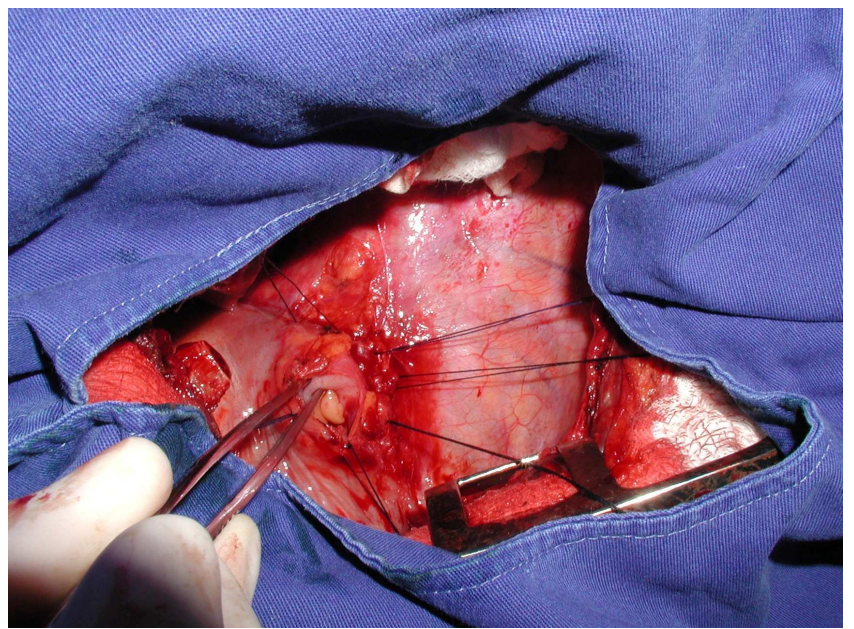

Figura 3 - Intra-operatório: saco herniário aberto com identificação do fundo gástrico já na cavidade abdominal. diafragmático de $5 \mathrm{~cm}$ de diâmetro com pontos separados de fio de algodão 2.0. Drenada a cavidade pleural sob visão direta, com síntese da parede torácica por planos com Poliglactina 910 1.0.

A análise histopatológica do material ressecado evidenciou malformação adenomatóide cística congênita do pulmão.

Paciente evoluiu com dor em hemitórax esquerdo no pós-operatório, controlada com analgesia oral com opióide, sem mais intercorrências. $\mathrm{O}$ dreno torácico foi removido no quarto dia e a paciente recebeu alta no sétimo dia de pósoperatório sem queixas, e sem complicações ou sintomas até um ano de pós-operatório.

\section{EMBRIOLOGIA}

O diafragma se origina do septo transverso na terceira semana de gestação, a partir do mesentério ventral da parede abdominal anterior. $\mathrm{O}$ septo transverso migra, posteriormente, e se une a porções do diafragma que se estendem lateralmente a partir do mesentério dorsal, adjacente ao mediastino e, posteriormente, a partir da parede abdominal, de miótomos que formam bandas musculares. Durante as primeiras semanas de vida embrionária, as vísceras abdominais e torácicas migram livremente por defeitos situados dos dois lados da coluna vertebral denominados hiatos ou canais pleuroperitoneais ${ }^{4}$.

Membranas pleuroperitoneais completam a porção posterior do diafragma, fechando estes hiatos, ou canais de Bochdalek, entre a sétima e nona semanas de gestação. A hérnia diafragmática congênita posterolateral consiste na persistência dos canais de Bochdalek (Figura 4), pelos quais a herniação de vísceras abdominais é o efeito lógico do aumento da pressão intra-abdominal ${ }^{4}$.

Apesar do epônimo, aparentemente a localização e a etiologia das hérnias descritas por Bochdalek não condizem com as aceitas atualmente.

A HB é mais comum à esquerda ocorrendo em $60 \mathrm{a}$ $80 \%$ dos $\operatorname{casos}^{4,6}$, ao que se atribui a obliteração mais precoce

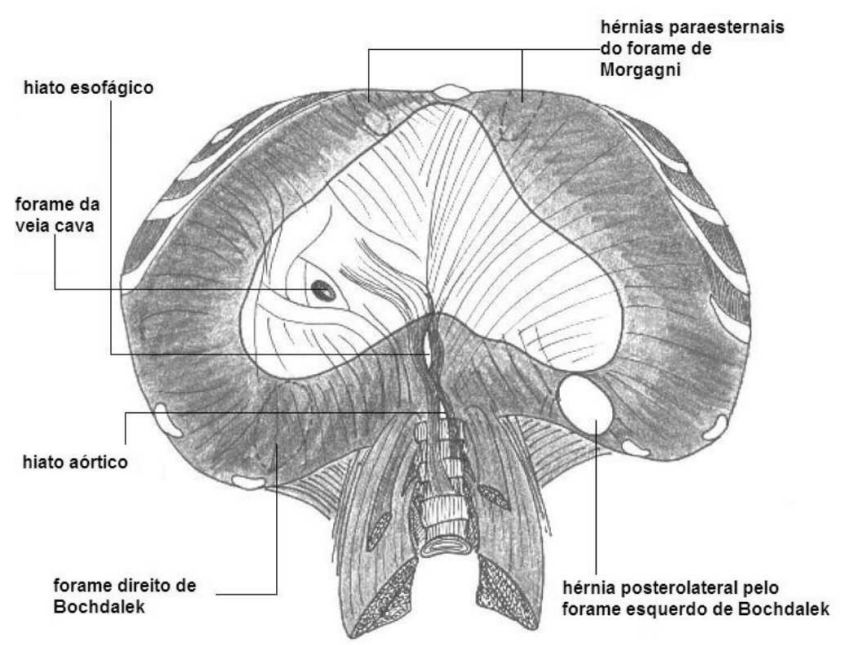

Figura 4 - Face abdominal do diafragma com seus orifícios anatômicos e os canais de Bochdalek e de Morgagni. 
do canal pleuroperitoneal direito e ao efeito de barreira causado pelo fígado.

São geralmente defeitos pequenos, sendo raros os casos de hérnias grandes ou bilaterais ( 3 a 6\%). Como explicação, tem-se o fato de que hérnias maiores ou bilaterais apresentem maior morbidade e mesmo incompatibilidade com a vida, restando ao diagnóstico no adulto, hérnias pequenas, capazes de permanecerem longos períodos assintomáticas. Notadamente a hérnia diafragmática congênita apresenta-se como malformação única, em contraposição à maioria dos outros defeitos congênitos, geralmente múltiplos.

\section{MALFORMAÇÕES ASSOCIADAS}

Em 26\% dos casos esporádicos, as hérnias de HB estão associadas a outras malformações congênitas, principalmente neurológicas (hidrocefalia, Arnold-Chiari) e cardiovasculares (hipoplasia pulmonar, patência do ducto arterioso, prolapso de valvas cardíacas $)^{10}$. Na forma familiar de HB, são mais freqüentes malformações genitourinárias que neurológicas. A hipoplasia pulmonar e a patência do ducto arterioso são as alterações mais usuais nas duas formas de HB. Um estudo reportou incidência de $26 \%$ de vícios de rotação em hérnias de Morgagni. Síndromes que apresentam risco associado de hérnias diafragmáticas congênitas incluem as Síndromes de Down, Turner, Prader-Willi e de Noonan ${ }^{11}$.

\section{MALFORMAÇÃO ADENOMATÓIDE CÍSTICA}

No caso apresentado, a paciente apresentava em associação à HB malformação adenomatóide cística (MAD). A MAD é uma malformação rara do conteúdo não-cartilagenoso das estruturas respiratórias terminais. Ela consiste em tecidos císticos e sólidos que, ao exame histológico, mostra, predominantemente, elementos bronquiolares. Pode apresentar-se de três formas: macrocística e microcística ou sólida. No tipo macrocístico de MAD, há o predomínio de um ou mais cistos grandes, enquanto que na forma microcística, ocorrem mais múltiplos pequenos cistos. No tipo sólido, prevalece massa de material sólido sem ar em seu interior. A lesão pode afetar, em parte ou na totalidade, um lobo pulmonar, por vezes dois lobos ou até todo o pulmão.

Em alguns casos, a MAD pode apresentar-se com graves sintomas respiratórios no nascimento. Lesões grandes podem causar compressão mediastinal ou do pulmão contra-lateral. Eventualmente, a MAD pode ser diagnóstico diferencial de neonatos com insuficiência respiratória aguda. São descritos casos de diagnóstico na infância tardia ou mesmo na adolescência. Até onde sabemos, este é o único caso descrito de malformação adenomatóide cística diagnosticada em adulto em associação com HB. O tratamento de escolha da MAD é a ressecção cirúrgica ${ }^{12}$.

\section{DIAGNÓSTICO}

Com possível envolvimento de diversos órgãos intraperitoneais e retro-peritoneais, a história clínica e o exame físi- co muitas vezes pobre ou inespecífico, trazem algumas dificuldades ao diagnóstico das hérnias diafragmáticas.

As vísceras mais comumente encontradas na HB à esquerda são o omento maior (em até 73\%), estômago (envolvido em $40 \%$ dos casos), intestino delgado, colo, e baço e gordura retroperitoneal, sendo raramente encontrados o rim, glândula supra-renal, ureter, fígado, apêndice e pâncreas ${ }^{1,7,13}$.

São descritos relatos fatais decorrentes de diagnóstico tardio ou errôneo e de complicações gastrointestinais, como encarceramento de pâncreas e adrenal ${ }^{13}$, ou morte súbita por desvio de mediastino e alteração do eixo cardíaco ${ }^{2}$. Não é infreqüente o erro diagnóstico, confundiddo-se particularmente com a pneumonia, que aparece em até $62 \%$ das séries de casos em crianças. O pneumotórax representa situação com maior potencial de morbidade, com relato de perfuração gástrica na inserção de dreno de tórax ${ }^{14}$.

As ocasionais hérnias diafragmáticas direitas apresentam o fígado, intestino delgado e colo e, mais raramente, a vesícula biliar e o rim herniados.

$\mathrm{Na}$ maioria dos casos verifica-se sem a presença de saco herniário. Este é formado por peritôneo e pleura parietal e ocorre dependendo do período de desenvolvimento embrionário no qual ocorreu o defeito. A herniação tardia pode ser precedida pelo início da formação das membranas pleuroperitoneais que, em sua fase inicial, apresentam poucas fibras musculares e invadem o tórax envolvendo as vísceras abdominais. A incidência de saco herniário varia de 10 a $38 \%{ }^{6}$. Alguns autores advogam que a sua presença pode postergar o aparecimento de sintomas e seu rompimento posterior pode desencadear sintomatologia tardia no adulto ${ }^{2}$.

A maioria dos casos diagnosticados em adultos são achados incidentais de exames. Pacientes sintomáticos apresentam-se, em grande parte, como emergência cirúrgica decorrente de complicações abdominais, embora autores relatem envolvimento de vísceras torácicas em até $67 \%$ dos ca$\operatorname{sos}^{15}$.

O estrangulamento com obstrução e sofrimento de vísceras ocas é o mais comum, podendo evoluir com perfuração e suas complicações infecciosas. Achados menos freqüentes como o sangramento digestivo, sintomas dispépticos decorrentes de herniações gástricas transitórias - caso apresentado neste relato - são descritos. Outras raras e graves intercorrências foram descritas como volvo gástrico, icterícia, herniação de cisto ovariano e fístula colo-pleural ${ }^{7}$.

Quando a herniação é transitória ou intermitente apresentam menor morbidade e permitem o preparo do paciente para cirurgia eletiva, como no caso que apresentamos.

\section{EXAMES SUBSIDIÁRIOS}

O diagnóstico pode ser obtido por exames de imagens do tórax, principalmente quando vísceras ocas ascendem à cavidade torácica. A radiografia de tórax pode mostrar apagamento do seio costofrênico, elevação da cúpula diafragmática e imagens aéreas ou hidro-aéreas no tórax. Algumas vezes podem ser definidas imagens de alças intestinais ou colônicas e mesmo sombra de vísceras parenquimatosas. O derrame pleural é associação comumente encontrada ${ }^{16}$. A 
utilização de sonda nasogástrica pode auxiliar o diagnóstico quando o estômago se encontra comprometido

Em um paciente com encarceramento de intestino delgado à direita foi feito com auxílio do RX diagnóstico inicial de Síndrome de Chilaiditi (rara anomalia que consiste na interposição da alça intestinal entre o fígado e o diafragma), o que demonstra as limitações do método ${ }^{13}$.

A tomografia computadorizada pode facilmente diagnosticar mesmo pequenas hérnias. A utilização de cortes finos, de 5 e $1 \mathrm{~mm}$, e a reconstrução em eixos axial, coronal e sagital reduz os erros de identificação, embora falsos positivos e falsos negativos ocorram ${ }^{6}$.

O diagnóstico definitivo pode ser obtido com exames contrastados, embora sejam contra-indicados quando há suspeita de intercorrências. A endoscopia no caso apresentado mostrou-se de grande valia no diagnóstico, sendo útil na avaliação de possíveis complicações gástricas.

\section{TRATAMENTO}

O tratamento das HB é cirúrgico e consiste na redução dos órgãos herniados e fechamento do orifício diafragmático, primário ou com auxílio de próteses sintéticas (principalmente em defeitos grandes ou com pouco tecido em seu limite posterior); as próteses constituem boa opção. Por ocasionar a exposição de órgãos intra-peritoneais à tela, é preferida a utilização das de polytetrafluoroetileno-PTFE-expandido (Gore-Tex Mycro Mesh; W.L. Gore \& Associates Inc., Flagstaff, AZ, U.S.A.) ${ }^{3,9}$, embora estudos experimentais evidenciem a formação de aderências, ainda que em menor incidência, com o uso desta e de próteses de polipropileno revestidas ${ }^{16}$. Estudo randomizado utilizando tais materiais em fechamento de grandes defeitos hiatais em 31 pacientes mostrou tratar-se de procedimento seguro, com menor índice de recorrência, confirmado por série de cinco casos de correção de hérnias recidivadas em crianças ${ }^{17}$.

Nas HB esquerdas, o tratamento clássico é pela via torácica, com justificativa de melhor acesso às estruturas pleuro-mediastinais, embora alguns autores descrevam a intervenção abdominal como igualmente satisfatória para lise de aderências ${ }^{1,7,9}$. A abordagem da cavidade peritoneal com formação de bridas e suas possíveis complicações talvez justifique a preferência pela via torácica. A utilização bem sucedida de acessos minimamente invasivos, abdominal e torácico, é descrita, embora o tratamento laparoscópico seja dificultado pela localização posterior do defeito ${ }^{3,18-20}$.

Nas HB à direita, o acesso torácico é preferido pela facilidade de exposição do diafragma, embora com adequada mobilização do lobo hepático esquerdo esta possa ser também obtida por via laparotômica.

A abordagem abdominal é mandatória nos casos de suspeita de complicações inerentes às suas vísceras, para adequada avaliação destas, ou nas situações onde exista a presença associada de vícios de rotação. Na vigência de tratamento utilizando-se a via torácica mas com necessidade de reabordagem por acesso abdominal decorrente de necrose e perfuração do estômago justifica este tipo de procedimento na dúvida diagnóstica9 ${ }^{9}$. Raramente há a necessidade de intervenção combinada toraco-abdominal.

No presente relato, como o estômago se apresentava sem complicações à endoscopia, optou-se pela toracotomia, por considerarmos esta abordagem de melhor facilidade e menor morbidade na correção do defeito nas hérnias diafragmáticas congênitas de apresentação tardia.

\begin{abstract}
Bochdalek's hernia is a congenital malformation of the posterolateral diaphragm region. It is more common on the left and more frequently seen in newborns and rare in adults, with over a few 100 reported cases. We present a case of Bochdalek's hernia in a 49-year-old patient with long term dyspeptic symptoms. The upper endoscopy showed a gastric fundus herniation sliding into the chest through the diaphragmatic defect. The patient also presented with a rare pulmonary malformation diagnosed during surgery. It was corrected through thoracic approach with no other complications (Rev. Col. Bras. Cir. 2008; 35(1): 040-044).
\end{abstract}

Key words: Hernia, diaphragmatic; Diaphragm; Hernia, Diaphragmatic /congenital.

\section{REFERÊNCIAS}

1. Ibañez-Fuentes JR, Nieto-Ocampo AE, Bermudez-Gimenez A, Olivares Aguirre A. Hernia de Bochdalek en el adulto. Gac Méd Méx 2003;139(1):69-72.

2. Salaçin S, Alper B, Cekin N, Gülmen MK. Bochdalek hernia in adulthood: a review and autopsy case report. J Forensic Sci. 1994;39(4): 1112-6.

3. Al-Emadi M, Helmy I, Nada MA, Al-Jaber H. Laparoscopic repair of Bochdalek hernia in an adult. Surg Laparosc Endosc Percutan Tech. 1999;9(6): 423-5.

4. Scheer CW, Linville JL. Congenital diaphragmatic hernia through foramen of Bochdalek. Arch Surg. 1965;91(5):823-8.
5. Vanamo K. A 45-year perspective of congenital diaphragmatic hernia. Br J Surg. 1996;83(12):1758-62.

6. Mullins ME, Stein J, Saini SS, Mueller PR. Prevalence of incidental Bochdalek's hernia in a large adult population. AJR Am J Roentgenol. 2001;177(2):363-6.

7. Karanikas ID, Dendrinos SS, Liakakos TD, Koufopoulos IP. Complications of congenital posterolateral diaphragmatic hernia in the adult. Report of two cases and literature review. J Cardiovasc Surg. 1994;35(6):555-8.

8. Habib E, Bellaïche G, Elhadad A. Complications de la hernie de Bochdalek méconnue de l'adulte. Revue de la littérature. Ann Chir. 2002; 127(3):208-14. 
9. Perch P, Houck WV, DeAnda A. Symptomatic Bochdalek hernia in an octogenarian. Ann Thorac Surg. 2002;73(4):1288-9.

10. Bujanda L, Larrucea I, Ramos F, Muñoz C, Sánchez A, Fernández I. Bochdalek's hernia in adults. J Clin Gastroenterol. 2001;32(2):155-7.

11. Singh S, Bhende MS, Kinnane JM. Delayed presentations of congenital diaphragmatic hernia. Pediatr Emerg Care. 2001;17(4):269-71.

12. Zach MS, Eber E. Adult outcome of congenital lower respiratory tract malformations. Thorax. 2001;56(1):65-72.

13. Mar Fan MJ, Coulson ML, Siu SK. Adult incarcerated rightsided Bochdalek hernia. Aust N Z J Surg. 1999;69(3):239-41.

14. Mei-Zahav M, Solomon M, Trachsel D, Langer JC. Bochdalek diaphragmatic hernia: not only a neonatal disease. Arch Dis Child. 2003;88(6):532-5.

15. Powers RC, Sejdinaj I, Oberschneider PB. Strangulated foramen of Bochdalek hernia in the adult. Am J Surg. 1966;111(5):749-51.

16. Marcondes W. Estudo dos efeitos das próteses de polipropileno revestidas com métodos de barreira implantadas na cavidade peritoneal de coelhos [dissertação]. São Paulo(SP): Universidade Federal de São Paulo, 2003.
17. Harinath G, Senapati PS, Pollitt MJ, Ammori BJ. Laparoscopic reduction of an acute gastric volvulus and repair of hernia of Bochdalek. Surg Laparosc Endosc Percutan Tech. 2002;12(3):180-3.

18. Losanoff JE, Sauter ER. Congenital posterolateral diaphragmatic hernia in an adult. Hernia. 2004;8(1):83-5. Epub 2003 Sep 20.

19. Silen ML, Canvaster DA, Kurkchubasche AG et al. Vídeoassisted thoracic surgical repair of a foramen of Bochdalek hernia. Ann Thorac Surg 1995; 60:448-50.

20. Rice ED, O’Boyle CJ, Colm J et al. Laparoscopic repair of Bochdalek hernia in an adult. ANZ J Surg 2001; 71(7): 443-45.

Como citar este artigo:

Oliveira DR, Rodrigues Jr AJ. Hérnia de Bochdalek em adulto. Revisão da literatura. Rev Col Bras Cir. [periódico na Internet] 2007; 34(6). Disponível em URL: http://www.scielo.br/rcbc

Endereço para correspondência:

Daniel Riccioppo C. F. de Oliveira

R.Dr. Barachisio Lisboa, 88

Vila Madalena

05441-090 - São Paulo - SP

E-mail: danriccioppo@uol.com.br 